\title{
A Learning Classes-Based No-Reference Image Quality Assessment Algorithm Using Natural Scenes Statistics
}

\author{
Moad El Abdi ${ }^{1(\mathbb{*})}$ and Zhenqi Han ${ }^{2}$ \\ ${ }^{1}$ School of Computer Engineering and Science, Shanghai University, \\ Shanghai 200444, China \\ moad.elabdi@gmail.com \\ ${ }^{2}$ Shanghai Advanced Research Institute, Chinese Academy of Sciences, \\ Shanghai 201012, China
}

\begin{abstract}
In this paper, we propose a new method for learning a No-Reference Image Quality Assessment that relies on the preparation of examples of different classes of distorted images. Our learning model learns classes of quality for each distortion type. This is achieved by preparing classes of training examples in a way that for each type of distortion, while we introduce a level of distortion to go from "high quality" to "low quality" care must be taken in that a lower rate (integer) is given to the new class for which the actual degree of the distortion have degraded the perceptual quality. A radial basis function (RBF) network is trained using some extracted perceptual features to separate the images into ten categories of quality. The same RBF reuses the same features to quantify a distorted test image by predicting its category of quality. Experimental results on the Distorted Face Database show that the method is effective.
\end{abstract}

Keywords: Image quality assessment - Generalized gaussian distribution · BLIINDS-II · Radial basis function network · Distorted face images

\section{Introduction}

In biometry, the assessment of the quality of input data is very important in the measure that it allows to improve the performance of a recognition system. Image quality issues such as Gaussian noise, blurring, and JPEG introduced during acquisition, transmission, and compression of images are examples of problems that can be faced by a biometric system. Since in most image-processing applications, human beings are the ultimate users, then the best way to evaluate the quality of an image is by means of a human subject. However, subjective evaluation is not practical in real-world applications since human observers are generally unavailable or expensive. Thus, there is a need for objective image quality assessment (IQA) algorithms that can automatically predict perceived image quality. Objective quality metrics are generally classified into 3 categories: (1) FR metrics (Full Reference), which compare the image to be evaluated to a model of reference of this one; (2) RR metrics (Reduced Reference), which compare a description of the image to be evaluated to a description of the model of reference; and (3) NR metrics (No Reference), which quantifies the quality of the image to be evaluated on the 
basis of some knowledge of this image (i.e., without the use of the model or the description of reference). Since original images are often inaccessible in practical applications, many researchers became interested in designing blind/No-Reference quality assessment algorithms. NR-IQA algorithms can also be divided into distortion-specific, and non-distortion-specific.

The majority of the existing NR metrics belongs to the former, meaning that they depend on the acquisition artifact (blocking, blur, ringing, etc.), thus since it is assumed that the distortion type is known, they only try to quantify severity. The limitation of these methods is that since they are distortion-specific thus they are application specific. The latter are independent from the distortion type and assume that training examples which possess the same or similar types of distortions as in testing images are available and use machine learning techniques, where features related to perceptual quality are extracted to train learning models for quality assessment. These approaches also follow one of the 2 trends: (1) training-based approaches; (2) natural scene statistics (NSS) based approaches. The former are as reliable as the features extracted to train the learning machine. The existing algorithms that adopt this approach often do not provide perceptual evidence to the features extracted. Algorithms that follow the latter approach are very promising since they have the advantage of relying on statistical modeling of features that are very representative of the perceptual quality.

In this paper, we propose a non-distortion-specific NR-IQA method, where examples of face images from the Distorted Face Database (DFD) [2, 3] are prepared in the form of classes of quality for a given distortion, and features related to image quality from a published metric (BLIINDS-II) [1] are extracted to train a RBF network by classifying the different classes of quality using the subjective class quality scores. These subjective scores are based on giving a lower rate to a class of images of a given distortion for which change in perceptual quality is noticed as long as a level of the distortion is introduced to the images. The quantification process is done by the same RBF model which will reuse the same extracted features for classifying a test image on the basis of the extent of belonging to these learned classes.

The rest of this paper is organized as follows. In Sect. 2, we review relevant literature on No-Reference image quality assessment measures. The proposed model for designing blind image quality assessment is discussed in Sect. 3. In Sect. 4 we describe the experimental setup and the results, respectively. We conclude the paper in Sect. 5.

\section{Previous Work}

Most present-day NR-IQA algorithms are distortion-specific which assume that the distortion type is known and only quantify severity. These algorithms quantify one or more distortions such as blockiness $[4,5]$, blur [6, 7] or ringing [8, 9] and score the image accordingly. And since they are distortion specific so they are application specific. Non-distortion-specific NR-IQA algorithms often assume that examples which possess the same or similar types of distortions as in testing images are available and apply machine learning techniques for quality assessment. The NR-IQA problem is usually transformed to a regression or classification problem, where a regressor or classifier is 
trained using features related to image quality. These approaches usually follow one of the following two trends: (1) training-based approaches and (2) NSS-based approaches.

\subsection{Training Based NR IQA Approaches}

These approaches rely on training a learning machine to predict the image quality score based on a number of features extracted from the image. The features used to train these models often do not have a perceptual justification for each feature. Support vector regression (SVR) [10] and neural networks [11] can be used to learn the mapping from feature space to subjective quality. For example in [12], the author presented a new approach for image quality assessment based on visual component detection and supervised machine learning. First, the input image is processed by a visual component detection module to identify relevant objects. Then, each detected visual component is processed by a quality assessment module, resulting in a numeric quality score for each detected visual component. And finally, the individual component scores are combined in a weighted sum, resulting in an overall quality score. Li et al. [11] developed a NR image quality assessment algorithm that deploys a general regression neural network (GRNN) which assess image quality by approximating a function relationship between the extracted features and subjective mean opinion scores. Ye and Doermann [13] proposed a NR IQA method which uses a visual codebook-based method for feature space quantization, and then learns the mapping from the quantized feature space to image quality score.

\subsection{NSS Based NR IQA Approaches}

Natural-Scene Statistics metrics relies on the assumption that natural images form a small subset in the space of all possible images, and measuring for a given distorted image the departure from the expected natural image statistics. These methods are promising but relies on extensive statistical modeling and reliable generalization of the models. The most state-of-art NSS-based NR algorithms are: (1) BLIINDS-II [1], (2) BIQI [14], (3) DIIVINE [15] (4) BRISQUE [16]; (5) NIQE [17] and DESIQUE [23].

In $[18,19]$, the authors used four degradation factors (contrast, distortion of structure, orientation anisotropy) in the DCT domain, without image modeling to build the BLIINDS index.

In BLIINDS- II, they have refined their approach by modeling image features using a generalized Gaussian distribution model which encompasses a wide range of behavior of distorted DCT coefficients. BIQI algorithm explore NSS-based wavelet and a number of 18 features are extracted for each image. BRISQUE uses scene statistics of locally normalized luminance coefficients to quantify possible losses of 'naturalness' in the image due to the presence of distortions. The features used derive from the empirical distribution of locally normalized luminances and products of locally normalized luminances under a spatial NSS model. DIIVINE is different from BIQI in that it is a NSSbased IQA concept developed by extracting a much richer set of features that capture the dependencies between subband coefficients over scales and orientations, as well as utilizing the perceptually relevant divisive normalization procedure. NIQE is based on 
the construction of a "quality aware" collection of statistical features based on a simple and successful space domain NSS model. These features are derived from statistical regularities of natural, undistorted images. And DESIQUE extracts log-derivative-based statistical features at two image scales in both the spatial and frequency domains, upon which a two stage framework is employed to evaluate quality.

\section{Algorithm}

Our method consists in the design of a No-Reference quality assessment algorithm for face images. Our work is based on the observations and conclusions of the authors on the work in [20]. In their extensive comparative study of seven well-known No-Reference image quality assessment algorithms (including BLIINDS-II), they observed that a same NR metric provided different values of correlation when one considers same distortions from different databases. In brief, the performance of each metric are dependent of the used database. This behavior is due to the fact that all metrics have been trained on LIVE database [21]. In other words, the metrics are sensitive to different datasets, thus, they have to be retrained when new training samples are available. Since our aim is to build a NoReference quality assessment algorithm for face images, we propose to train one of those metrics, namely BLIINDS-II, in an extensive face images database: Distorted Face Database [2, 3].

In our approach for designing the No-Reference quality assessment algorithm, we propose a method based on learning some classes of quality to model the quality metric. To achieve this goal, we prepare some examples to compose many classes for each type of distortion: from "high quality" to "low quality" (i.e. (class 1 , classe2, .., class 10) for Blur; and similarly for Noise and JPEG). Then each of the classes will be given a rate of quality by a human expert. Care must be taken in the process of preparing the classes in a way that when introducing a certain degree of a type of distortion to the examples to go from higher class to lower class, difference in perceptual quality must be observed by the expert who is performing the experiment in order to give a lower score to the new low quality class for which a change in perceptual quality have been noticed. Thus, it is as if we have a subjective class quality assessment model based on human-rated classes of distorted images. The rates (labels) are based on the idea of how perceptual quality changes as the different levels of a given distortion are introduced. In the other hand, we use an NSS-based NR quality metric BLIINDS-II to extract features that are representative to perceptual quality, the features are chosen based on how their statistics vary as the image quality changes [1]. Then, we perform feature extraction for the different prepared classes of one type of distortion (e.g. Blur), and an RBF network is trained to classify the images into one of the ten classes using the 10 human labels. The quality score of a test image is given by the extent to which it belongs to these ten classes.

\subsection{Feature Extraction}

In this paper, we use the features extracted to build the BLIINDS-II algorithm. This algorithm employs the notion of natural scenes statistics. The main idea of this approach 
is based on the hypothesis that the functions of the Human Visual System has evolved in function of time and are adapted to the statistics of the world where the human being evolves. BLIINDS-II algorithm is based on the computation of features formed from the estimated parameters of a NSS model of the image DCT coefficients in different spatial resolutions. The DCT features are chosen based on the observation that their statistics change as the image quality changes. The DCT computation is applied to $5 \times 5$ blocks with a 2-pixel overlap between the blocks. In [1], the authors have modeled image features using a generalized Gaussian distribution model which encompasses a wide range of behavior of distorted DCT coefficients. The generalized Gaussian density is given by:

$$
f(x \mid \alpha, \beta, \gamma)=\alpha e^{-(\beta|x-\mu|)^{\gamma}}
$$

Where $\mu$ is the mean, $\gamma$ is the shape parameter, and $\alpha$ and $\beta$ are the normalizing and scale parameters. The parameters of the model are then utilized to extract features for quality prediction. The 4 extracted (over 3 scales) model-based features are:

Generalized Gaussian Model Shape Parameter. The generalized Gaussian model of the non-DC DCT coefficients from $5 \times 5$ blocks is deployed. The shape parameter feature $\gamma$ is calculated over all block in the image, then two ways pooling process is performed for the scores. First, by computing the lowest $10^{\text {th }}$ percentile average local block shape scores $(\gamma)$ across the image, and second by computing the $100^{\text {th }}$ percentile average of the local $(\gamma)$ scores across the image.

Coefficient of Frequency Variation. The coefficient of frequency variation feature is given by

$$
\zeta=\sqrt{\frac{\Gamma(1 / \gamma) \Gamma(3 / \gamma)}{\Gamma^{2}(2 / \gamma)}-1}
$$

Where $\Gamma$ is the gamma function. The computation of the feature $\zeta$ is done for all $5 \times 5$ blocks in the image. Then a pooling process is conducted for $\zeta$ across the image by averaging over the highest $10^{\text {th }} \%$ and over all $100^{\text {th }} \%$ of the local block scores.

Energy Subband Ratio Measure. The radial spatial frequencies of the DCT coefficients increase when moving the top-left corner to the bottom-right corner. Thus three frequency bands $n=1,2,3$ are defined. The average energy in frequency band $n$ is defined as the model variance $\sigma_{n}^{2}$ corresponding to band $n$

$$
E_{n}=\sigma_{n}^{2}
$$

To obtain the mean of $R_{2}$ and $R_{3}$, the ratio $R_{n}$ is computed for $n=2,3$

$$
R_{n}=\frac{\left|E_{n}-\frac{1}{n-1} \sum_{j<n} E_{j}\right|}{E_{n}+\frac{1}{n-1} \sum_{j<n} E_{j}}
$$


Where $E_{n}$ the average energy in frequency is band $n$ and $\frac{1}{n-1} \sum_{j<n} E_{j}$ is the average energy up to frequency band $n$. This feature is computed for all $5 \times 5$ blocks in the image, and the pooling is done in the same way as for $\zeta$.

Orientation Model-Based Feature. Ageneralized Gaussian model is fitted within each region in the block, and the variance of $\zeta$ is computed along each of the three orientations of the DCT coefficients. The scores are pooled as before across all $5 \times 5$ blocks in the image (highest $10^{\text {th }} \%$ and $100^{\text {th }} \%$ averages).

\subsection{RBF Network Model}

The radial basis function network (RBF network) is a multilayer network composed of 3 layers. Neurons of the hidden layer do react significantly to only a narrow part of the input space in function of a radial activation function (Gaussian in general) in a manner analogous to the behavior of cells of the cerebellum [22].

The output of the network is simply the linear combination of the RBF neurons outputs multiplied by their respective connection weights. Four parameters are to be set in a RBF network:

- The number of RBF neurons (number of neurons in the unique hidden layer).

- The position of the centers of Gaussians of each neuron.

- The width of these Gaussians.

- The connection weights between the RBF neurons and the output neuron(s).

Any modification in these parameters directly results in a change of network behavior. The number of RBF neurons and the position of the Gaussians are two parameters intimately related. Two options are available to us:

Either $\mathrm{I}$ is not too big and then $\mathrm{N}=\mathrm{I}$;

Either I is too big then we choose $\mathrm{N} \ll \mathrm{I}$ (with $\mathrm{I}$, the number of elements in the training set).

Choosing of the Centers Position with $\mathbf{N} \ll \mathbf{I}$. Afirst method consists in randomly choosing the centroids from the examples presented to the network. This approach is not bad since statistically the centroids will be representative of the distribution of examples. But this technique is not optimal either, because it is possible to choose very poorly placed centroids. Another solution, allowing to obtain best results, is to select the centroids via vector quantization (Learning Vector Quantization: LVQ). This solution will allow to achieve the best allocation of centroids as possible. Many also propose to apply a self-organizing Kohonen map in order to select the centers. In this work we will be using a self-organized learning mechanism to estimates the locations of the centers of the RBF and this is done by K-means clustering algorithm.

In K-means clustering, initial centers are chosen, and the input patterns are fed oneby-one. The RBF neurons will be competing with each other and only the winner neuron is moved closer to the first pattern. In the next iteration, another training pattern is fed for the next epoch and so on. The steps of the algorithm are [24]: 
Initialization. Suppose $\mathrm{M}$ is number of chosen $\mathrm{RBF}(\mathrm{s})$ centers to be in the hidden layer denoted by $\overrightarrow{t_{k}}$ with $k=1,2, \ldots, M$. Different random values of initial centers $\overrightarrow{t_{k}}(0)$ are generated with " 0 " referring to the first iteration.

Sampling. A sample vector from the input space: $\overrightarrow{X(n)}$ for iteration " $\mathrm{n}$ " is drawn.

Similarity Matching. Let $\overrightarrow{k(X)}$ denote the index of the best matching (winning) center of input vector $\vec{X}$.

$$
\vec{k}(X)=\arg \min _{k} \vec{X}(n)-\overrightarrow{t_{k}}(n), \mathrm{k}=1,2, \ldots, \mathrm{M}
$$

The computation of the above minimum for all competing centers will return the index of the winning center for the pattern $\vec{X}$. This is done by taking the "arg" of this distance.

Updating. The center of the RBF is adjusted according to the updating rule:

$$
\overrightarrow{t_{k}}(n+1)=\left\{\begin{array}{l}
\overrightarrow{t_{k}}(n+1)+\eta\left[\vec{X}(n)-\overrightarrow{t_{k}}(n)\right], k=k(\vec{X}) \\
\overrightarrow{t_{k}}(n), \text { otherwise }
\end{array}\right.
$$

Continuation. Increment $\mathrm{n}$ by 1 and go back to step 2 . The iteration process is stopped when no noticeable changes are observed in the centers $\vec{t}_{k}$.

Selecting Beta Values. Once the centers $t_{j}$ are computed, the next step in the training process of the RBF network is to determine the width $\beta$ of the Gaussians. Since k-means clustering is used to select the centers, then one simple method for specifying the Beta coefficients is to set sigma (the standard deviation) equal to the average distance between all points in the cluster and the cluster center.

$$
\sigma_{j}=\frac{1}{N} \sum_{i=1}^{N} X_{i}-t_{j}, \quad j=1,2, \ldots, M
$$

where $t_{j}$ is the cluster centroid, $\mathrm{N}$ is the number of training samples belonging to this cluster, and $X_{i}$ is the ith training sample in the cluster.

Beta coefficients can be calculated using the obtained $\sigma_{j}$

$$
\beta_{j}=\frac{1}{2 \sigma_{j}^{2}} \quad j=1,2, \ldots, M
$$

Output Weights. The final set of parameters to train are the weight of each of the connections (RBF-output). These can be trained using gradient descent (also known as least mean squares).

This computation can be described by the following matrix equation: 


$$
\left[\begin{array}{ccc}
\mathrm{G}\left(\mathrm{X}_{1}-\mathrm{t}_{1}^{2}\right) & \ldots & \mathrm{G}\left(\mathrm{X}_{1}-\mathrm{t}_{\mathrm{M}}^{2}\right) \\
\mathrm{G}\left(\mathrm{X}_{2}-\mathrm{t}_{1}^{2}\right) & \ldots & \mathrm{G}\left(\mathrm{X}_{2}-\mathrm{t}_{\mathrm{M}}^{2}\right) \\
\vdots & \ldots & \vdots \\
\mathrm{G}\left(\mathrm{X}_{\mathrm{N}}-\mathrm{t}_{1}^{2}\right) & \ldots & \mathrm{G}\left(\mathrm{X}_{\mathrm{N}}-\mathrm{t}_{\mathrm{M}}^{2}\right)
\end{array}\right]\left[\begin{array}{c}
\mathrm{w}_{1} \\
\mathrm{w}_{2} \\
\vdots \\
\mathrm{w}_{\mathrm{M}}
\end{array}\right]=\left[\begin{array}{c}
\mathrm{y}_{1} \\
\mathrm{y}_{2} \\
\vdots \\
\mathrm{y}_{\mathrm{N}}
\end{array}\right]
$$

With $G\left(X_{i}-t_{j}^{2}\right)=\exp \left(-\frac{1}{2 \sigma_{j}^{2}} X_{i}-t_{j}^{2}\right)$ the Gaussian, The elements of the column matrix $\mathrm{Y}$ are the desired outputs, $\mathrm{M}$ the number of centers and $\mathrm{N}$ the number of samples in the training set.

Finally, once the learning is done, the test part can begin. It also can be summarized in a matrix equation:

$$
\left[\begin{array}{lll}
G\left(X_{i}-t_{1}^{2}\right) & \ldots & G\left(X_{i}-t_{M}^{2}\right)
\end{array}\right]\left[\begin{array}{c}
w_{1} \\
w_{2} \\
\vdots \\
w_{M}
\end{array}\right]=S
$$

Where $X_{i}$ here is a test pattern and $\mathrm{S}$ is the test output.

In this paper, the aforementioned RBF model will be used to classify the distorted face images into 10 categories of quality. The quantification process is reduced to a classification problem and the machine quality score $(\mathrm{ms})$ of a distorted image is equal to the class of quality to which it belongs where, Class 10 correspond to the best quality and Class 1 correspond to the worst quality.

\section{Experiments}

In order to evaluate the performances of the method, we use face images from the Distorted Face Database. 500 images for each distortion (blur. Gaussian noise, JPEG) are prepared for training purpose. The 500 face images affected with blur contain 10 classes of quality from 10 to 1 (50 images for each class). The RBF model will learn how to classify the distorted face images into 10 categories of quality. The same process is performed for 500 face images affected with Gaussian noise and 500 face images affected with JPEG.

We use both self-validation and cross validation tests in the experiment. In self validation the 500 face images affected with a distortion (blur. Gaussian noise or JPEG) are used in both training and testing, while in a cross validation, 150 other distorted (with blur, GN, JPEG respectively) images are used for testing.

We compare the machine image quality score "ms" generated by the classifier with the corresponding human labeled class quality score "hs". Specifically, we compute the difference between the two scores $|\mathrm{ms}-\mathrm{hs}|$ and analyze its statistics over the testing images. Note that both "ms" (generated from the classifier) and "hs" are integers in the scale 1 to 10. In Tables 1, 2 and 3, the distribution is shown in cumulative statistics [12], for example, in the cross validation row of Table 1, " $65.3 \%$ " in column " 0 " and " $97.3 \%$ " in column " 1 " means that on $65.3 \%$ and on $97.3 \%$ of the testing images affected with blur, the difference Ims-hsl is equal to 0 , and 1 respectively. Therefore, we can remark 
that the classifier generates machine scores close to those from a human expert even for cross validation.

Table 1. Quantitative system performance for Blur.

\begin{tabular}{l|l|l|l}
\hline \multirow{2}{*}{ Blur } & \multicolumn{3}{|c}{ Distribution of difference } \\
\cline { 2 - 4 } & 0 & 1 & 2 \\
\hline Self-validation & $87.8 \%$ & $99.6 \%$ & $100 \%$ \\
\hline Cross validation & $65.3 \%$ & $97.3 \%$ & $99.3 \%$ \\
\hline
\end{tabular}

Table 2. Quantitative system performance for noise.

\begin{tabular}{l|l|l|l}
\hline \multirow{2}{*}{ Noise } & \multicolumn{3}{|c}{ Distribution of difference } \\
\cline { 2 - 4 } & 0 & 1 & 2 \\
\hline Self-validation & $80 \%$ & $97.4 \%$ & $99.6 \%$ \\
\hline Cross validation & $58.7 \%$ & $96 \%$ & $98.7 \%$ \\
\hline
\end{tabular}

Table 3. Quantitative system performance for JPEG.

\begin{tabular}{l|l|l|l}
\hline \multirow{2}{*}{ JPEG } & \multicolumn{3}{|c}{ Distribution of difference } \\
\cline { 2 - 4 } & 0 & 1 & 2 \\
\hline Self-validation & $71.6 \%$ & $94.8 \%$ & $99.8 \%$ \\
\hline Cross validation & $46.7 \%$ & $89.3 \%$ & $98.7 \%$ \\
\hline
\end{tabular}

Tables 2 and 3 show the distribution of the same difference for images affected with Gaussian noise and JPEG respectively.

\section{Conclusion}

We present an approach for No Reference image quality assessment based on some features formed from the estimated parameters of a NSS model of the image DCT coefficients and a learning method of classes of quality. In our method, we first prepare some examples to compose 10 classes of quality in an appropriate manner (a low rate is given to a class of examples when degradation in perceptual quality is observed), and a classifier is trained on these 10 classes using the extracted perceptual features. The quantification process is reduced to a classification problem and the quality score of a distorted test image is equal to the class of quality to which it belongs. Experiments on face images altered with three types of distortions (Blur, Noise, and JPEG) yielded encouraging results. 
Acknowledgment. Project supported by Shanghai Science and Technology Committee. The No. of grand is 13511503200 .

\section{References}

1. Saad, M.A., Li, C., Bovik, A.C.: Blind image quality assessment: a natural scene statistics approach in the DCT Domain. IEEE Trans. Image Process. 21(8), 3339-3352 (2011)

2. Gunasekar, S., Ghosh, J., Bovik, A.C.: Face detection on distorted images using perceptual quality-aware features. Proc. SPIE-IS\&T Electr. Imag. 9014, 90141E (2014)

3. www.ideal.ece.utexas.edu//suriya/DFD/

4. Muijs, R., Kirenko, I.: A no-reference blocking artifact measure for adaptive video processing. In: European Signal Processing Conference (Eusipco) (2005)

5. Wang, Z., Bovik, A.C., Evans, B.L.: Blind measurement of blocking artifacts in images. Proc. IEEE Int. Conf. Image Proc. 3, 981-984 (2000)

6. ParvezSazzad, Z.M., Kawayoke, Y., Horita, Y.: No reference image quality assessment for jpeg2000 Based on spatial features. Signal Process. Image Commun. 23(4), 257-268 (2008)

7. Ramin, N.: Versunemétrique sans référence de la qualitéspatiale d'un signal vidéodans un contexte Multimedia. Ph.D. thesis, Université de Nantes (2009)

8. Hantao, L., Klomp, N., Heynderickx, I.: A no reference metric for perceived ringing artifacts in Images. IEEE Trans. Circuits Syst. Video Technol. 20(4), 529-539 (2010)

9. Barland, R., Saadane, A.: Blind quality metric using a perceptual map for JPEG2000 compressed images. In: International Conference on Image Processing (ICIP) (2006)

10. Tang, H., Joshi, N., Kapoor, A.: Learning a blind measure of perceptual image quality. In: Proceedings of IEEE Conference Computer Vision Pattern Recognition, pp. 305-312 (2011)

11. Li, C., Bovik, A.C., Wu, X.: Blind image quality assessment using a general regression neural network. IEEE Trans. Neural Netw. 22(5), 793-799 (May 2011)

12. Luo, H.: A training-based no-reference image quality assessment algorithm. In: International Conference on Image Processing (ICIP), pp. 2973-2976 (2004)

13. Ye, P., Doermann, D.: No-reference image quality assessment based on visual codebook. In: Proceedings of the IEEE International Conference on Image Process, pp. 3089-3092 (2011)

14. Moorthy, A.K., Bovik, A.C.: A two-stage framework for blind image quality assessment. In: ICIP, pp. 2481-2484 (2010)

15. Moorthy, A.K., Bovik, A.C.: Blind image quality assessment: From natural scene statistics to perceptual quality. IEEE Trans. Image Process. 20(12), 3350-3364 (2011)

16. Mittal, A., Moorthy, A.K., Bovik, A.C.: No-reference image quality assessment in the spatial domain. IEEE Trans. Image Process. 20, 3350-3364 (2012)

17. Mittal, A., Soundararajan, R., Bovik, A.C.: Making a completely blind image quality analyzer. IEEE Signal Process. Lett. 22(3), 209-212 (2013)

18. Saad, M., Bovik, A.C., Charrier, C.: A DCT statistics-based blind image quality index. IEEE Signal Process. Lett. 17(6), 583-586 (2010)

19. Saad, M.A., Bovik, A.C., Charrier, C.: DCT Statistics approach to no- reference image quality assessment. In: Proceeding of 2010 IEEE 17th ICIP, pp. 313-316 (2010)

20. Nouri, A., Charrier, C., Saadane, A., Maloigne, C.F.: A statistical comparison of no-reference image quality assessment algorithms. In: Color and Visual Computing Symposium, IEEE (2013)

21. http://live.ece.utexas.edu/research/quality

22. Haykin, S.: Neural Networks: A Comprehensive Foundation, 2nd Edn. Prentice Hall (1999) ISBN: 0-13-273350-1 
23. Zhang, Y., Chandler, D.M.: An algorithm for no-reference image quality assessment based on log-derivative statistics of natural scenes. Proc. SPIE-IS\&T Electr. Imaging 8653, 86530J (2013)

24. Mittal, A., Soundararajan, R., Bovik, A.C.: Making a completely blind image quality analyzer. IEEE Signal Process. Lett. 22(3), 209-212 (2013) 